\title{
Intracranial hemorrhage during aeromedical transport and correlation with high altitude adaptations in the brain
}

This article was published in the following Dove Press journal:

Open Access Emergency Medicine

6 December 2012

Number of times this article has been viewed

\section{Timur Kouliev' \\ Airron Richardson ${ }^{2}$ \\ Cai Glushak ${ }^{3,4}$}

'Beijing United Family Hospital, Beijing, People's Republic of China;

${ }^{2}$ Department of Emergency Medicine,

The Methodist Hospitals, Gary, IN,

${ }^{3}$ AXA Assistance USA, Chicago, IL;

${ }^{4}$ Section of Emergency Medicine,

University of Chicago, Chicago,

IL, USA
Correspondence: Timur Kouliev Beijing United Family Hospital, \#2 Jiangtai Lui, Beijing, I000I6, People's Republic of China

Tel +86 I0 59277120

Email timur.kouliev@ufh.com.cn
Abstract: Aeromedical transport is challenging not only because of limitations of equipment, unfamiliar surroundings, and challenging environmental conditions, but also due to difficulty in developing methodologies for research and data collection. To our knowledge, neurological changes at the oxygen tensions of a pressurized cabin have not been systematically studied. Here we report a case of intracranial hemorrhage during aeromedical transport and review the body's cardiovascular and respiratory adaptation to decreased ambient oxygen tension. Previous experience with high altitude cerebral edema serves as guidance for mitigating the effects of vasogenic edema in patients at risk of neurological events who travel by air. Review of this case and relevant altitude-related physiological changes may be grounds for more conservative recommendations on aeromedical transport after an acute neurological event.

Keywords: pediatric, disaster, triage, equity, pregnancy, survival

\section{Introduction}

Medical care in the setting of aeromedical transport is challenging in part due to limitations of equipment, unfamiliar surroundings, and changing environmental conditions. The complexity of familiar medical emergencies is significantly increased in comparison with those occurring in the emergency department or intensive care unit. Although vibration, noise, patient and caregiver stress, prolonged sitting, and sleeplessness all contribute to physiological adaptation, the most important and unique difference is the body's cardiovascular and respiratory adaptation to decreased ambient oxygen tension, leading to travel in a relatively hypoxic environment.

A typical aircraft cabin is pressurized to $8000 \mathrm{feet}$, with the resulting oxygen tensions commonly sufficiently low to cause severe desaturations in patients with chronic cardiac or respiratory disease, and in those with acute respiratory infection. To our knowledge, neurological changes at the oxygen tensions of a pressurized cabin have not been systematically studied. However, a small number of articles have looked at the neurological complications suffered by individuals living at altitude and those rapidly ascending to high altitude. Although ascent in commercial aircraft is not effectively as high as in these studies, the unifying concept is the decrease in partial pressure of oxygen and resulting adaptive mechanisms, especially in a central nervous system that is recovering from an acute event or injury.

It is through research on acute mountain sickness that we have learned about the brain's adaptation to altitude. Despite the rapidly growing experiences of aeromedical providers, few experiences from altitude medicine have been transferred into 
knowledge of neurological safety in the air. The goal of this review is to be able to advise appropriately traveling patients with active or recovering illness, the physicians who accompany them, and those who clear them to fly. After we present a case of cerebral hemorrhage recurrence during aeromedical transport, we systematically review the literature relating to central nervous system adaptations at altitude, both in aircraft and on mountain expeditions, and examine future directions of research to keep the brain safe in the air.

\section{Case report}

A 53-year-old previously healthy male complained of nausea and severe headache an hour after landing, awaiting a connecting flight. The patient's wife noted that he was not acting like himself and dialed 911 for transport to the nearest emergency department. A right cerebellar lobar hemorrhage with mass effect was diagnosed and evacuated on an emergency basis. Postoperatively, the patient did well and was discharged on day 7 . He only complained of mild dysmetria on the right side. The etiology of the hemorrhage was not clear. There was no elevation of blood pressure, no history of anticoagulant or antiplatelet drug use, and findings on neurological imaging and surgical exploration did not suggest arteriovenous malformation or tumor. He was cleared to fly on day 10 in agreement with current guidelines, ${ }^{1}$ accompanied by a medical attendant, in a commercial aircraft.

On the day of travel, the patient denied headache, dizziness, vertigo, nausea, chest pain, or shortness of breath. He denied a history of smoking, drinking alcohol, or drug use. His family history was noncontributory. His discharge medications included metoprolol $50 \mathrm{mg}$ daily and famotidine $20 \mathrm{mg}$ twice daily. His vitals were blood pressure 130/88 $\mathrm{mmHg}$, heart rate 75 beats per minute, respiratory rate 15 per minute, and oxygen saturation $98 \%$ on room air. Cardiovascular, respiratory, and abdominal examinations were unremarkable. Mild dysmetria was noted on finger to nose examination on the right, unchanged from before. The patient ambulated without assistance, and the rest of the neurological examination was unremarkable. The patient was taken to the airport via emergency medical services without incident and transported in a wheelchair from the entrance to the gate for comfort. He refused food and read a magazine comfortably until departure. After takeoff, the patient slept, denied symptoms, and his vital signs remained unchanged for the 4 hours of flight.

Twenty minutes before landing, the patient complained of nausea and generalized 5/10 headache. Blood pressure readings were $140 / 70 \mathrm{mmHg}$ and $160 / 90 \mathrm{mmHg}$, pulse
80 per minute, respiratory rate 22 , and oxygen saturation $96 \%$. At the time of landing, the headache decreased, and the patient had an episode of emesis. The blood pressure was $140 / 75 \mathrm{mmHg}$, pulse 86 beats per minute, respiratory rate 20 per minute, and oxygen saturation $96 \%$ on room air. Neurological examination revealed that he was alert and oriented, the cranial nerves were intact, and that he had normal strength and sensation in both upper and lower extremities. There was a marked increase in dysmetria on the right side, and his gait was wide and unstable with a positive Romberg's sign. He was transported to the nearest emergency department as an emergency, where a new hemorrhage was diagnosed in the right cerebellar hemisphere within the cavity of the previously evacuated hematoma. There was no mass effect. The patient was admitted to the neurosurgical intensive care unit, treated expectantly, and observed to recover without incident to his previous functional status. Once again, no cause was discovered for the repeat hemorrhage.

\section{Discussion}

The majority of research on altitude-related illness has concentrated on the pulmonary and cardiovascular effects of decreased oxygen tension. ${ }^{2}$ Basic neurovascular physiology research and clinical studies in neurology have focused heavily on high altitude cerebral edema, a condition characterized by reversible vasogenic regional cerebral edema of the deep white matter and increased blood-brain barrier permeability. ${ }^{3}$ These changes in the blood-brain barrier are thought to result from hypoxia-induced overperfusion of microvascular beds, leading to increased release of inflammatory factors and mediators of capillary leakage, such as vascular endothelial growth factor and nitric oxide. ${ }^{4}$

High altitude cerebral edema is clinically often considered to be an extension of acute mountain sickness, and is characterized by changes in consciousness with different degrees of psychiatric disturbances, ataxia, and confusion that may lead to coma and death in previously healthy individuals. ${ }^{3}$ It is unclear to what degree the pathophysiology of high altitude cerebral edema can be applied to aircraft transports, where "effective altitudes" are lower due to cabin pressurization. A few studies suggest that there may be a connection. Recent magnetic resonance imaging studies have suggested that on ascent to high altitudes, all people develop swelling of the brain to some extent. However, it is not known if the swelling is due to increased volume of blood in vessels or swelling of the brain itself. ${ }^{5}$ Individuals who move to higher altitude appear to have a higher risk of ischemic stroke. Jha and Anand studied Indian military personnel stationed at high 
altitude for a period of 2 years. In that study, a significantly increased risk of ischemic stroke was demonstrated, which the authors postulated was due to polycythemia. ${ }^{6}$

No study has evaluated the influence of high altitude on the frequency of intracranial hemorrhage. Some experts do not recommend ascent to altitude for patients who have had lobar hemorrhage that resulted from amyloid angiopathy, but that recommendation is entirely due to a high risk of recurrence, and the recommendations are not based on an increased recurrence at altitude. ${ }^{7}$ The influence of high altitude on the bleeding risk of cerebral aneurysms, arteriovenous malformations, and cavernous hemangiomas is unclear. At cabin pressure, it is also not known if predictable changes in blood pressure occur and to what extent the cerebral blood flow increases or predisposes to bleeding.

It is entirely possible that our patient's repeat hemorrhage at altitude was coincidental. However, it is notable that the above studies on hypoxia are unanimous in the concern about increasing blood flow and augmenting capillary permeability in the setting of altitude-related relative hypoxia. Potentially important is also that the patient's intracranial bleeding occurred not immediately upon pressurization or depressurization of the cabin, but after 4 hours of relative hypoxia, or in the case of the first hemorrhage, after hours of being on the ground.

\section{Conclusion}

This report may have an impact on recommendations for travel after an acute neurological event. The current aeromedical travel guidelines have no specific recommendation regarding travel after intracerebral hemorrhage, but state the following regarding neurological events in general "... [ the patient is to be] observed until sufficient time has passed to assure stability of the neurological condition. Clearly, the risk of post-event complications, the physical and mental disability, and the decreased capacity to withstand the stresses of flight are cogent reasons not to fly. Once the acute phase of recovery is over and the patient is stable, travel may be reconsidered."1

If the observations in this report are supported, they may influence more conservative recommendations for aeromedical transport of patients recovering from surgical interventions, those with brain tumors or arteriovenous malformations, or any others who might be at risk of complications from capillary leak. Without pertinent data, proper advice will need to be based on opinion of experts and must be individualized based on the specifics of the case and the patient's acceptance of unquantifiable risk. Additional research is needed to elucidate the pathophysiology of hemorrhagic stroke at altitude and consequent means to prevent such events. In the meantime, a more conservative approach and longer recovery time may be recommended prior to commercial air transport, given the circumstances reported.

\section{Disclosure}

The authors report no conflicts of interest in this work.

\section{References}

1. Medical Guidelines for Airline Travel, 2nd ed., Aerospace Medical Association Medical Guidelines Task Force. Aviat Space Environ Med. 2003 May;74(5 Suppl):A1-19.

2. Baumgartner RW, Siegel AM, Hackett PH. Going high with preexisting neurological conditions. High Alt Med Biol. 2007;8:108-116.

3. Basnyat B, Murdoch DR. High altitude illness. Lancet. 2003;361: 1967-1974.

4. Gallagher SA, Hackett PH. High altitude illness. Emerg Med Clin North Am. 2004;22:329-355.

5. Mórocz IA, Zientara GP. Volumetric quantification of brain swelling after hypobaric hypoxia exposure. Exp Neurol. 2001;168:96-104.

6. Jha SK, Anand AC. Stroke at high altitude: Indian experience. High Alt Med Biol. 2002;3:21-27.

7. Hackett PH, Roach RC. High altitude illness. N Engl J Med. 2001;345: 107-114.

\section{Publish your work in this journal}

Open Access Emergency Medicine is an international, peer-reviewed, open access journal publishing original research, reports, editorials, reviews and commentaries on all aspects of emergency medicine. The manuscript management system is completely online and includes a very quick and fair peer-review system, which is all easy to use.

\section{Dovepress}

Visit http://www.dovepress.com/testimonials.php to read real quotes from published authors. 